This document is published in:

Journal of Ambient Intelligence and Smart Environments (2014), 6 (2), 237-255.

DOI: http://www.dx.doi.org/10.3233/AIS-140255

(C) 2014 IOS Press 


\title{
An approach to develop intelligent learning environments by means of immersive virtual worlds
}

\author{
David Griol $^{\text {a, }}$, José Manuel Molina ${ }^{\text {a }}$ and Zoraida Callejas ${ }^{b}$ \\ ${ }^{a}$ Department of Computer Science, Carlos III University of Madrid, Avda. de la Universidad, 30, 28911, \\ Leganés, Spain \\ E-mail: \{david.griol,josemanuel.molina\}@uc3m.es \\ ${ }^{\mathrm{b}}$ Department of Languages and Computer Systems, University of Granada, CITIC-UGR, C/Pdta, Daniel Saucedo \\ Aranda s/n, 18071, Granada, Spain \\ E-mail: zoraida@ugr.es
}

Keywords: Intelligent Environments, immersive virtual worlds, e-learning, conversational agents, Second Life, OpenSimulator, Sloodle, speech interaction

\begin{abstract}
Merging Immersive Virtual Environments, Natural Language Processing and Artificial Intelligence techniques provides a number of advantages to develop Intelligent Environments for multiple applications. This paper is focused on the application of these technologies to develop intelligent learning environments. Education is one of the most interesting applications of immersive virtual environments, as their flexibility can be exploited in order to create heterogeneous groups from all over the world who can collaborate synchronously in different virtual spaces. We highlight the potential of virtual worlds as an educative tool and propose a model to create learning environments within Second Life or OpenSimulator combining the Moodle learning management system, embodied conversational metabots, and programmable 3D objects. Our proposal has been applied in several subjects of the Computer Science degree in the Carlos III University of Madrid. The results of the evaluation show that developed learning environment fosters engagement and collaboration and helps students to better understand complex concepts.
\end{abstract}

\section{Introduction}

Research work in the area of Intelligent Environments aims to design places that can adapt, respond to, support and enhance human activities transcending the limits of direct human perception [7,30,43]. Most of existing intelligent environment research has focused on the design of system architectures to manage communication between hardware devices and sensors in physical spaces. However, Immersive Virtual Environments (IVEs) $[5,8,39]$ make it possible to extend this work as a powerful testbed for Artificial Intelligence technologies in industrial domains, intelligent assistance in e-commerce on the web, computer games industry, applications which up to now have used 2D simulations (e.g., evacuation of buildings in emergencies or traffic control), robotics, or even advanced mixed reality intelligent environments [16].

Virtual Worlds are already in common use as tools for researching intelligent environments and other aspects of pervasive computer science. These virtual environments or "metaverses" are computer-simulated graphic environments in which humans cohabit with other users through their avatars. Traditionally, virtual worlds have had a predefined structure and fixed tasks that the user could carry out. However, social virtual worlds have emerged to emphasize the role of social interaction in these environments, allowing the users to determine their own experiences and supporting interactions (such as remote collaboration, adaptive and 
intelligent environments) that are not readily available in physical environments.

This way, in the three-dimensionality context it is very appropriate to develop virtual robots with the same appearance as that of the human-driven avatars. These new virtual robots are called metabots, term coined from the contraction of the terms metaverse and robot. Metabots can have the same appearance and capabilities of the avatars for human users, thus intensifying the perception of the virtual world, providing gestures, glances, facial expressions and movements necessary for the communication process.

Thanks to the social potential of virtual worlds, the development of metabots has become attractive for institutions, companies and researchers interested in human-machine communication. However, social interaction in virtual worlds is usually carried out using only text by means of chat-type services. In order to enhance communication in these environments, we propose the integration of conversational agents to develop intelligent metabots with the ability of oral communication and, at the same time, which benefit from the visual modalities provided by virtual worlds.

A conversational agent $[24,33,40,49]$ can be defined as an automatic system able of emulating a human being in a dialogue with another person, in order to complete a specific task. Two main objectives are fulfilled thanks to its use. The first objective is to facilitate human-machine interaction using speech. The second one is to make information and functionalities accessible for users with motor and visual disabilities, as they avoid the use of traditional interfaces, such as keyboard and mouse.

With the growing maturity of conversational technologies, the possibilities for integrating conversation and discourse in intelligent learning environments are receiving greater attention [26,44]. Conversational agents have been developed to meet a wide range of applications in education, including tutoring [50], question-answering [58], conversation practice for language learners [18], pedagogical agents and learning companions [15], dialogue applications for computeraided speech therapy with different language pathologies [57], and dialogues to promote reflection and metacognitive skills [31].

Our paper focuses on three key points. Firstly, we promote the use of open source applications and tools for the creation of educative intelligent environments in virtual worlds, such as the tools and applications provided by means of the combination of the Open-
Simulator virtual worlds ${ }^{1}$ and the Moodle learning management system (Module Object-Oriented Dynamic Learning Environment ${ }^{2}$ ). Secondly, we emphasize the benefits of working in immersive environments to create visual objects that can clarify concepts that are difficult to understand due to their abstraction level. Thirdly, we show that it is possible to successfully integrate speech technologies and natural language processing in virtual worlds and show a practical application of the integration and evaluation of these functionalities to create an educative environment in the Second Life virtual world. ${ }^{3}$

The remainder of the paper is structured as follows. Section 2 presents the potential of virtual worlds as an educative tool and describes the main experiences carried out recently in the application of these technologies to develop intelligent environments. In Section 3 we center on Second Life and OpenSimulator, which are the most extended virtual worlds, and pay special attention to the specific resources it provides for educative purposes. Section 4 presents our proposal to develop rich learning environments in virtual worlds based on the integration of Sloodle (Simulation Linked Object Oriented Dynamic Learning Environment ${ }^{4}$ ), conversational metabots and manageable $3 \mathrm{D}$ learning objects. One of the main contributions consists of a new methodology for creating conversational metabots and improve speech interaction in virtual worlds. Section 5 describes an implementation of our proposal for several courses in the University Carlos III of Madrid, which evaluation is discussed in Section 6. Finally, in Section 7 we present the conclusions derived from our work and propose future work guidelines.

\section{Virtual worlds and intelligent learning environments}

Immersive Virtual Worlds provide a number of advantages for the development of intelligent environments [19]. As software, they are portable and so can be used in/from multiple locations. They can be quickly adapted to suit an experiment or demonstration and reduce the time and costs required to modify the real environment. Virtual worlds also allow creat-

\footnotetext{
${ }^{1}$ http://opensimulator.org

${ }^{2} \mathrm{http}: / /$ moodle.org/

${ }^{3}$ http://secondlife.com/

${ }^{4}$ http://www.sloodle.org/
} 
ing multiple instances that can be run simultaneously to compare the effects of different technologies, agents or initial conditions. They are also not limited to the constraints of the physical environment and can show information that would be otherwise invisible. They can be used not only as a way to prototype some processes and methods that in the real world take a long time (weeks, months, or even years), but also to slow time down and allow the observation of processes that in the real world occur too fast for human perception.

In addition, virtual worlds provide a combination of simulation tools, sense of immersion and opportunities for communication and collaboration that have a great potential for their application in education. However, as criticized in [21], many of the existing educative experiences in virtual worlds only replicate traditional approaches into the new environment, such as for example recreating classrooms co-located in a virtual world.

Although such direct translation does not leverage all the potential of the technology, it provides an added value in the case of online courses, for example, as a mean of e-assessment for large groups during a course [46], or as a mean to recreate face-to-face contact with classmates and teachers. Also they help to reduce costs and to offer improved learning activities to the students with the same budget. For example, Andrade et al. used avatar-mediated training in medicine to teach the students how to deliver bad news [2]. Usually, this is carried out hiring actors who play the role of standardized patients, which is very expensive and also limits the situations that the actors can portray. Avatars can be customized to create a wider range of scenarios and create a richer learning experience.

In these cases, when virtual worlds are employed to replicate real world activities, it has been reported that they have a similar effectiveness in terms of learning than in real world [27]. This happens mainly because, according to [55], the use of simulations that are close to reality allows students to "learn by doing" through real life situations, using role-playing scenarios along with the appropriate teaching methods $[1,6,22,51,56]$. Nevertheless, field experience is usually needed to obtain more meaningful learning. For example, Winn et al. evaluated the oceanographic knowledge obtained in a virtual environment as well as with real visits to the ocean and found that field experience was very important to contextualize learning for students with little prior experience in the topic [59].

The described situations do not take full advantage of all the possibilities that virtual environments offer, as virtual worlds benefit from unique characteristics that traditional learning environments do not present and provide opportunities for atypical ways of learning. For example, Ellison and Matthews used Second Life to teach eighteenth-century culture to students in an island that resembled the eighteenth-century London in which they had to reconstruct spaces with interactive elements with as much historical authenticity as possible and interact within them [17].

Also, virtual worlds allow creating manageable 3D representations of abstract entities and thus help students to construct mental models by direct observation and experimentation. Mikropoulos and Natsis identified that science and technology courses had much more presence in virtual worlds than social studies and argued that this might be because the concepts explained are usually more abstract, unobservable or far from everyday experience [42]. Thus, such topics can benefit to a greater extent form the representative capabilities of virtual worlds in order to train students in subjects that require spatial abilities and high order thinking skills.

For example, Limniou et al. provided virtual representations of molecules and chemical reactions, which only exist in a microscopic level and thus are far from the student's everyday experiences [37]. Similarly, Anthamatten and Ziegler employed a 3D representation of geographic formations using the GeoWall system. This allowed the students to see the correspondence between 2D topographic maps and 3D surfaces and interpret features such as slopes, ridges and valleys that are difficult to envision in 2D [4]. In the two experiences, virtual representations were created using a technology different from virtual worlds and the teacher operated the virtual model, which could not be manipulated by the students.

Other interesting unique characteristics of virtual worlds that were not exploited in these cases are immersion, manipulability and first order experience. In some virtual environments students are immersed in the virtual world, this provides enhanced interactivity that would be very difficult in traditional classrooms and that makes the students protagonists of the learning experience [9]. For example, Bakas and Mikropoulos report the use of a virtual spacecraft controlled by the students in an astronomy class in primary school [11]. The students usually had problems to overcome previous misconceptions of the planetary phenomena such as the relative distances and sizes of celestial bodies and the movements they describe. Within the virtual world, the students could approach 
the Earth in their aircrafts and have a situated perspective of the phenomena obtaining a first order experience of the concepts learned.

Such a sense of immersion generally fosters engagement of the students with the tasks and the course, or, in a more general sense, with the formation of their own understanding. According to [42], most of the educative studies of virtual worlds claim that virtual interaction favours engagement. However, other studies have reported that the virtual world sometimes distracts the attention of the students because of the novelty of the environment, or because of the interruption of avatars of other users of the virtual world who are not students of the subject. Some authors have taken advantage of these "distractions" to encourage the students to be more explorative. For example, Barab et al. employed a virtual world to make the students appreciate the complexities of real world problems by exploring different perspectives over the same problem [12]. Concretely, the students discovered that environmental awareness involves balancing ethical, economic, political and scientific factors.

Virtual worlds also provide new opportunities for collaboration, as they facilitate synchronous and asynchronous communication, supporting and enhancing student-student and teacher-student interaction. Petrakou reported that the students involved in their research socialized just like they do in real world courses, trying to get to know each other, and also learning to cope with the new environment [48]. He also reported that the student-student interaction continued after the course when some students discussed the assignments and tried to reach a common understanding of them.

Although virtual worlds can be employed as a tool for collaboration and communication to supplement face-to-face communication, the learning curve of using the virtual world may constitute a disadvantage [3] However, when used for multi-cultural and distributed learning, it can provide important advantages. For example, Kanematsu et al. report an experience with US, Korean and Japanese students for which they incorporated a language grid to Second Life that allowed multilingual discussion [29]. This way, virtual worlds can foster cooperative learning where students help each other; thus helping to eliminate conflicts among students with different cultural backgrounds.

Also the possibility to communicate with classmates embodied in their avatars can help to develop understanding of civic values and empathy. Park described the use of Second Life to make the students experience role playing with different ethnicities and gender roles, which allowed them to better recognize characteristics of male/female gender roles and give them a different perspective [45].

To fully benefit from all the described characteristics of the virtual worlds, the course contents must be active, project-based and designed following a pedagogical strategy that leverages the unique potential that virtual worlds offer [27]. According to [21], social constructivist pedagogies are more appropriate to design effective learning experiences in virtual worlds. This way, knowledge is acquired on the basis of authentic real-world problems, for which the solution is a communal task that must be achieved as the union of the individual efforts in the virtual environment.

Also competence in technologies and in particular in the use of the virtual environment has been found to be fundamental. As reported in [48], when the use of the virtual world is a new experience for the students, this technology itself constitutes a collaborative learning activity that contributes to socialization between students. Thus, Petrakou highlights that the teacher must be aware of the fact that the students need to familiarize with the virtual world before it is possible for them to focus on the actual course content [48]. For example, Jarmon et al. required as a condition to get a permission to register in the subject, that students had to meet some pre-class Second Life requirements, set up their accounts, create their avatars, and complete an online tutorial [27].

Some authors have addressed the challenge of identifying relevant features that provide a positive effect on learning and collaboration. These features are related to many factors such as the characteristics and usability of the virtual world, experience of the interaction (performance and satisfaction), learning and psychological factors (e.g. learning styles), and other characteristics of the students (e.g. age, or cultural background). A comprehensive study can be found in [35].

The benefits of virtual worlds for teaching and learning have fostered different research projects which aim is to help to use virtual environments in education. For example, the AVATAR Project (Added Value of teAching in a virtuAl woRld) [54] improves the quality of teaching and education in secondary schools through an innovative learning environment using a virtual world. The NIFLAR Project (Networked Interaction in Foreign Language Acquisition and Research) [28] is aimed at enriching, innovating and improving the learning process by using video conferencing and virtual worlds for interaction among students 
from Spain and Holland. V-LeaF (Virtual LEArning platForm) [52] is an educative platform developed by Universidad Autónoma de Madrid, which promoted cooperative and collaborative learning versus traditional learning.

Another project, 3D Learning Experiences Services ${ }^{5}$ included several educative projects for language learning developed with OpenSimulator for secondary education in the Netherlands. Similarly, the AVALON Project (Access to Virtual and Action Learning live Online) was aimed to language teachers and learners. ${ }^{6}$ The project sought to develop best practices in teaching and learning of languages in multi-user environments (MUVEs) like Second Life or OpenSimulator.

The +Spaces project (Positive Spaces - Policy Simulation in Virtual Spaces) [19] explored how virtual world technologies can be used to develop from polling and debating applications to more advanced role-playing simulation spaces. They concluded that role-playing is a useful tool for engaging learners in the complexities of real-world issues, often generating insights, which would not be possible using more conventional techniques.

A detailed literature review for studies that employ IVEs to conduct experimental studies in health care communication can be found in [47]. In this area, immersive environments have been used with the main aim of developing tools to train medical, nursing, pharmacy and other professional students in clinical skills and communication. The River City Project [32] simulated a city besieged by health problems. Students were organized in small research groups trying to find why residents were getting sick, using technology to track clues and figure out the causes of the disease, developing and testing hypotheses through experiments and extracting conclusions from the collected data. Additional projects illustrate key methodological advantages conferred by IVEs, including, ability to maintain simultaneously high experimental control and realism, manipulate variables in new ways, and unique behavioural measurement opportunities [47].

At elementary level, the Vertex Project [10] involved students from 9 to 11 years old, who worked in groups to combine traditional activities such as writing a story, draw a scene or make a collage with the plan and design of their virtual worlds, with the educative goal of developing their creativity and imagination. They ob-

\footnotetext{
${ }^{5}$ http://www.3dles.com/en/

${ }^{6} \mathrm{http} / / /$ avalonlearning.eu/
}

served that within a multi-user world, students improve their communication skills, learn to cooperate and improve their self-esteem and confidence.

Additionally, WiloStar3 $\mathrm{D}^{7}$ is a virtual school that offers distance education using virtual worlds to improve reading comprehension, problem-solving ability, and creativity. Students learn to experience and interact in the virtual world, and take different roles, according to the activity, participating in media projects with other students.

Also some projects have worked on creating virtual communities to share knowledge. For example, the Impending Gale was a project developed by Game Environment Applying Real Skills (GEARS) [13]. They created an online community called the Social and Educational Virtual World (VSEW) that contains avatars, chat, Voice Over Internet Protocol (VoIP) communication, areas for tutoring with teachers and various social objects that can interact with avatars. In a similar way, Euroland [36] was a project based on collaborative activities carried out among several classrooms in the Netherlands and Italy, which implied the creation of a 3D virtual world that was initially empty and was progressively populated with "culture houses".

However, although the pedagogical background has been carefully developed for these projects, the lack of interaction modalities in these environments may have a negative impact on the students' learning outcomes. Very recently, Mikropoulos and Natsis presented a tenyear review on the educative applications of virtual reality covering more than 50 research studies, and have pointed out that, although virtual worlds support multisensory interaction channels, visual representations predominate [42]. Unfortunately, there are a number of barriers that limit user interaction with computers when interfaces are only visual, as the users must have at least a minimum training for using the devices (mouse and keyboard) and must not be handicapped by visual or motor disabilities in order to use them.

In order to address these limitations, an alternative is to use conversational agents, which are designed to engage users in a conversation that aims to be as similar as possible as that between humans. Speech offers a greater speed for transmitting information, allows carrying out simultaneous tasks (liberating the user from the need to use his hands and/or eyes), informs about the identity of the speaker and allows disabled users to choose the modality that best fits them

${ }^{7}$ http://www.wilostar3d.com 
to interact with the computer. Also they have demonstrated to provide a more natural interaction than traditional GUI-based interfaces, and have a more affordable learning curve for people without enough technical knowledge [41]. As it will be described in Section 4 , we propose a methodology to develop conversational metabots in Second Life and also in virtual worlds generated with OpenSimulator. Our approach extends the typical architecture described for spoken dialogue systems with the visual modalities provided by 3D immersive environments and merges it with the multimedia possibilities of Sloodle and the pedagogical potential of 3D learning objects that provide a manageable representation of abstract entities.

\section{The Second Life and OpenSimulator virtual worlds}

Second Life (SL) is a three dimensional virtual world developed by Linden Lab in 2003 and accessible via the Internet. A free client program called the Second Life Viewer enables its users, called "residents", to interact with each other through motional avatars, providing an advanced level of a social network service.

Second Life has shown to be appropriate to build educative experiences, as it provides the possibility to create groups and communities to share activities and interests with other Second Life users. Residents can explore, meet other residents, socialize, participate in individual and group activities, create and trade items (virtual property) and services from one another. The stated goal is to create a user-defined world of general use in which people can interact, play, do business, and otherwise communicate.

SL is currently being used with success as a platform for education by many institutions, such as colleges, universities, libraries and government entities (e.g. Ohio University, Universidad Pública de Navarra, Cervantes Institute, Carlos III University of Madrid, etc.). Figure 1 shows an image of the Carlos III University of Madrid campus in Second Life.

Usually, these communities inhabit an island or "sim", which aspect and constructions can be continuously changed to recreate different spaces that can resemble the real world or be totally non-realistic. Within these spaces, communication between avatars can be carried out by means of gestures, voice, chat, videos, presentations, images and gestures of the

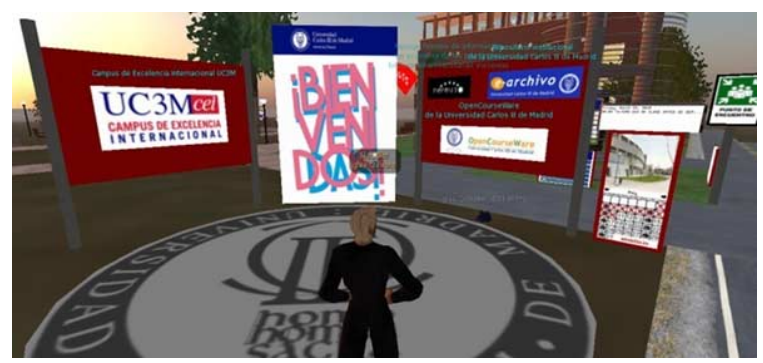

Fig. 1. Carlos III University of Madrid in Second Life.

avatars. This way, sims in SL can be filled with different types of multimedia information that can be shared, such as PDF documents, video, audio, webs and images.

In addition, it is possible to develop scripts for Second Life using the Linden Scripting Language (LSL) [53]. LSL [53] is a language oriented to state machines that allows controlling primitives, objects, and avatars by both physical events (touch, collide, listen) and logical events (temporizer, sensors). LSL includes a set of built-in functions, events and states definition and handling, different types and operators, and structures for flow control. ${ }^{8}$ Before their usage, the $3 \mathrm{D}$ objects are designed, developed and stored in the metabot's inventory. The llRezAtRoot function allows extracting the object and places it in SL. Then, the objects wait for commands that activate them and modify their behaviour.

Despite its interesting multimedia communication capabilities, speech communication is seldom employed in SL between avatars and metabots. Usually, metabots only provide information to the users, and thus the communication is unidirectional. In the cases in which a dialogue takes place between human users and automatic metabots, it occurs through the chat box interface. Thus, although spoken communication is technically plausible in Second Life, speech communication is optional: it can be activated or deactivated by the users, and the owners control the possibility to use speech in their respective lands.

We decided to use Second Life as a testbed for our research for several reasons. Firstly, because it is one of the most popular social virtual worlds available: its population is nowadays of millions of residents from around the world. Secondly, because it uses a sophisticated physics engine that generates very realistic simulations including collision detection, vehicle dynamics

${ }^{8}$ http://wiki.secondlife.com/wiki/LSL_Portal 


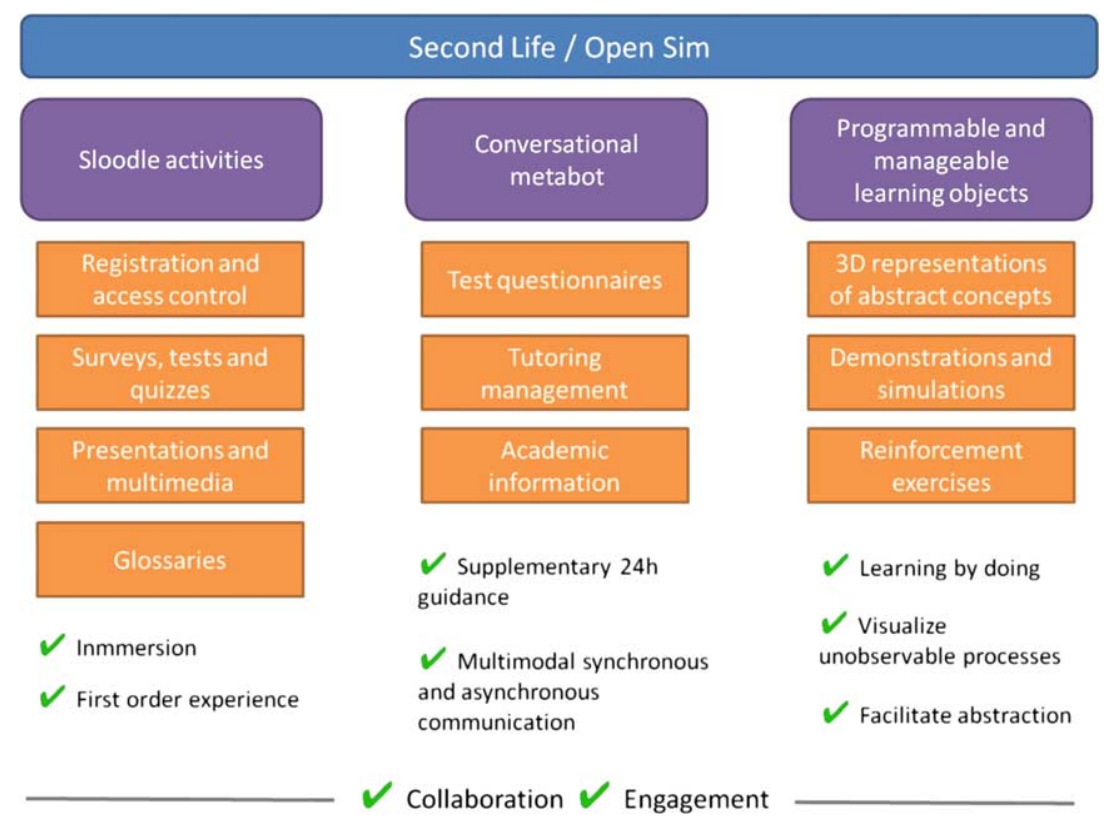

Fig. 2. Main components and advantages of our proposal for developing intelligent learning environments.

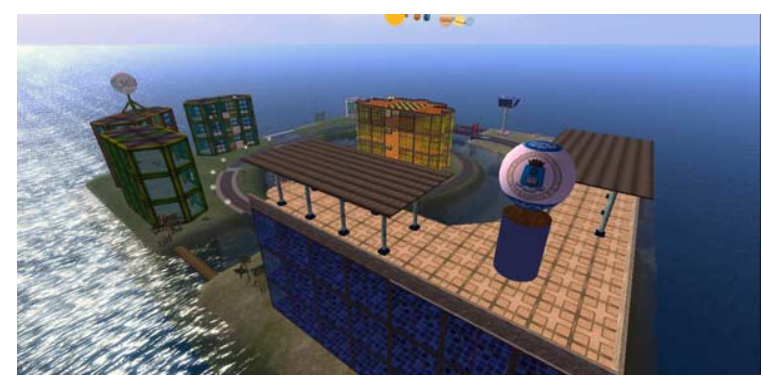

Fig. 3. The TESIS Island in Second Life.

and animation look $\&$ feel, thus making the avatars and the environment more credible and similar to the real world. Thirdly, because SL's capacity for customization is extensive and encourages user innovation and participation, which increases the naturalness of the interactions that take place in the virtual world. We own an island in Second Life called TESIS (see Fig. 3), in which we built its virtual facilities in which numerous educative activities are performed.

OpenSimulator (OpenSim) is an open-source alternative that can be used to simulate virtual environments similar to Second Life. It uses the same standard to communicate with their users and it is compliant with the Second Life viewer as well as a range of other viewers being developed by the open source community. Additionally, it allows to link completely free virtual worlds developed and hosted by different users us- ing technologies such as OsGrid. ${ }^{9}$ Although Second Life has been selected for the practical application of our proposal, the different components that will be described in the following section can also be employed in OpenSim with the main benefits of open-source and free.

\section{Our proposal to develop intelligent learning environments in immersive virtual worlds}

In order to exploit Second Life and OpenSimulator technologies to develop a fully equipped virtual environment for collaborative learning, we propose to develop educative experiences over the virtual world with three main components: Sloodle activities, an academic conversational metabot, and manageable learning 3D objects. These three components, their corresponding functionalities and main advantages are represented in Fig. 2.

Besides the features described in Section 3, Second Life presents several utilities specifically tailored for their free use in education; probably the most relevant is Sloodle, an open source project that integrates Second Life with the Moodle learning-management system. Moodle is a Course Management System (CMS), which makes possible the creation of virtual courses,

${ }^{9}$ http://www.osgrid.org/ 

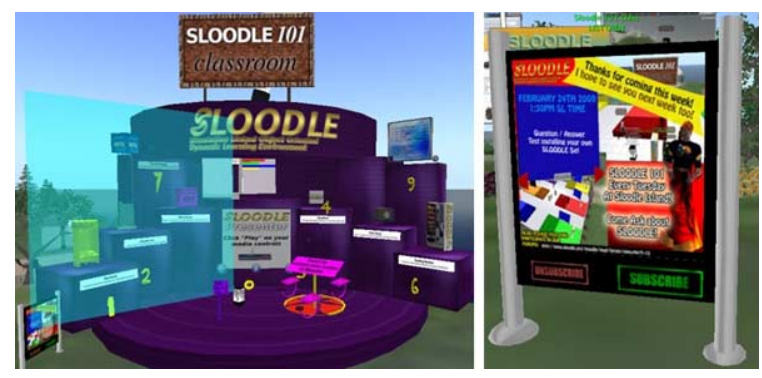

Fig. 4. Sloodle sim in Second Life.
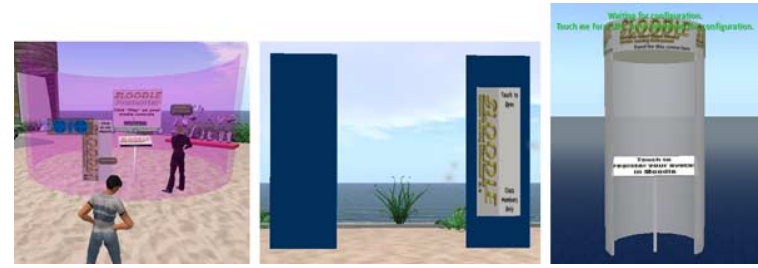

Fig. 5. Sloodle Access Checker activated by entering a login zone (left), Access Checker with a door (middle) and Enrol_Booth (right).

deliver exercises, provide forums, carry out exams, and all the tasks necessary for e-learning. Its integration with Second Life makes possible the access all these functionalities in the virtual world by means of an avatar, participating in classes and conferences in real time. Figure 4 shows a snapshot of a Sloodle course.

Along with Moodle facilities, and in order to make them more visual and attractive, Sloodle provides a range of tools for supporting learning and teaching to the immersive virtual world [23]. Firstly, it allows controlling the user registration and participation in a course thanks to an access checker as depicted in Fig. 5. Also new users can register in a course using the so-called Enrol_Booth.

Secondly, there are several tools to create surveys in Sloodle, such as Choice Horizontal, Quiz Chair or Quiz Pile on Fig. 6. Choice horizontal allows instructors to create and show surveys in the virtual world, compile the information and show the results in a course. With Quiz Chair an avatar can answer questionnaires of a course in SL, while Quiz Pile On provides a similar functionality with a more amusing format. In Quiz Pile On, questions float over a pile, and the students must seat over the correct answer, otherwise they falls over.

Thirdly, the Sloodle Presenter tool (see Fig. 7) allows creating presentations in Second Life, which can combine images, web pages and videos and may be configured so that any avatar or only the owner of the
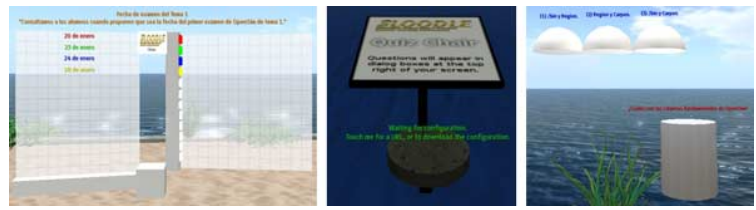

Fig. 6. Choice Horizontal, Quiz Chair and Quiz Pile On.

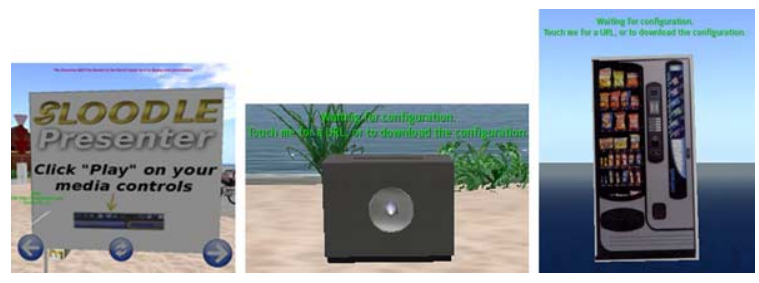

Fig. 7. PrimDrop (left) and Vending Machine (right).

corresponding sim controls the display of the presentation. Finally, there are other interesting tools for object sharing such as PrimDrop, which allows students to deliver their works by sending objects in Second Life, or the Vending Machine, which can be used to deliver objects to the students (Fig. 7).

Sloodle activities make it possible to create and control the collaborative sessions. The main objective in our proposal is to take advantage of the Moodle learning management system for the creation of virtual courses and the possibility of using these functionalities through the described 3D tools. In addition, the different activities requiring the Sloodle tools have been designed to make the participants be able to collaborate and express their ideas and make decisions, with the help of the material provided, such as documents, meeting minutes, presentations, and so on. This can be achieved thanks to the possibilities for user control and multimedia resources location in Sloodle. With the aim to coordinate and solve possible conflicts during collaboration, we propose to create several collaborative areas in the virtual campus, providing avatars with automatically generated annotations about the collaborative activity. This way, the environment enables also to control meetings through interactive ad-hoc developed objects. Each student uses this space to synchronously work with the other members of the team.

With regard the second component of our proposal, Fig. 8 shows the new architecture that we have developed for the integration of conversational metabots both in the Second Life and OpenSimulator-based virtual worlds. The conversational agent that governs the metabot is outside the virtual world, using exter- 


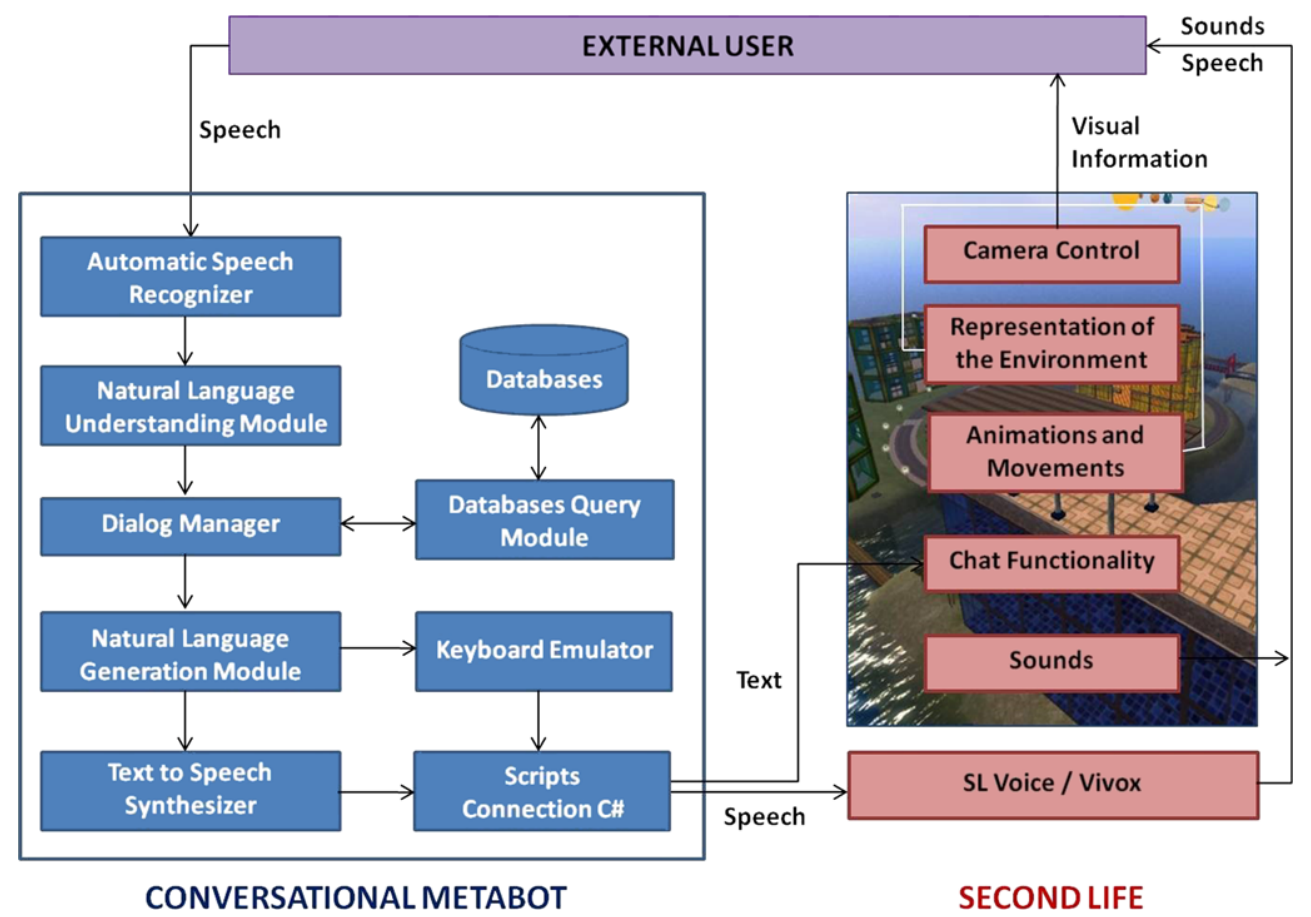

Fig. 8. Architecture defined for the development of conversational metabot for the interaction in virtual worlds.

nal servers that provide data access, language understanding, dialogue management, and speech recognition and synthesis functionalities. The developed conversational metabot makes it possible for the students to have permanent additional support in their study, and helps the teachers to leverage their workload in tasks like academic information providing, as well as automatizing evaluations by posing questionnaires and tests through the metabot.

Using this architecture user's utterances can be easily recognized, the transcription of these utterances can be transcribed in the chat in Second Life, and the result of the user's query can be communicated using both text and speech modalities.

Speech recognition and synthesis are performed using the Microsoft Speech Application Programming Interface (SAPI), integrated into the Microsoft Windows 7 operating system. We have also integrated modules for semantic understanding and dialogue management [25], which are based on speech grammars following the W3C SRGS specification ${ }^{10}$ and Voice Extensible Markup Language (VoiceXML) files, ${ }^{11}$ the standard defined by the W3C for speech ac-

\footnotetext{
${ }^{10}$ http://www.w3.org/TR/speech-grammar/

${ }^{11}$ http://www.w3.org/TR/voicexml20/
}

cess to Internet information and services. VoiceXML files are specified as conversational finite state machines in which users are in a conversational state, or dialogue, at a time. Each dialogue determines the next dialogue to transit by means of URIs. Execution is terminated when a dialogue does not specify a successor, or if it has an element that explicitly exits the conversation. The speech signal provided by the text to speech synthesizer is captured and transmitted to the voice server module in Second Life (SLVoice) using code developed in Visual C\#. NET and the SpeechLib library. This module is external to the client program used to display the virtual world and is based on the Vivox technology, which uses the RTP, SIP, OpenAL, TinyXPath, OpenSSL and libcurl protocols to transmit voice data. We also use the lipsynch utility provided by Second Life to synchronize the voice signal with the lip movements of the avatar. To enable the interaction with the conversational in Spanish using the chat in Second Life, we have integrated synthetic voices developed by Loquendo TTS. ${ }^{12}$

In addition, we have integrated a keyboard emulator that allows the transmission of the text transcription generated by the conversational avatar directly to

\footnotetext{
${ }^{12} \mathrm{http} / / / \mathrm{www} \cdot$ loquendo.com/
} 
the chat in Second Life. The system connection with the virtual world is carried out using the libOpenMetaverse library. This .Net library, based on the ClientServer paradigm, allows accessing and creating threedimensional virtual worlds, and it is used to communicate with servers that control the virtual world of Second Life.

Regarding the third component of our proposal, manageable learning objects can be constructed and placed in the environment to be manipulated by the students in order to clarify abstract concepts and allow them to "learn by doing". Second Life supports a parametric modelling method by providing a set of basic 3D models (cubes, spheres, triangular prisms, and so on). These models can be adjusted by designers to make more complex shapes by selecting geometric primitives and manipulating their parameters. The geometric features of the design can be adjusted within the world at any time, and object permissions can be changed to permit collaborative manipulation of a single object by multiple designers.

Our proposal is to use the Linden Scripting Language to create objects that react to the user inputs by changing their properties (position, volume, colour, name, description, owner, etc.) or specific behaviour. There are different tools that facilitate the use of this language. For instance, Scratch ${ }^{13}$ is a graphical programming language based on constructing programs by snapping together graphical blocks, which was developed by the Lifelong Kindergarten group at the MIT Media Lab. Scratch can be installed and freely redistributed and its source code is available under a license that allows modifications for non-commercial uses. This way, it is possible to create custom objects and applications.

\section{Intelligent learning environment developed for Second Life}

In this section we describe our experience developing a learning environment following the guidelines proposed in Section 4. We have used SL for creating a virtual learning environment supporting synchronous and collaborative learning at the Computer Science Department of the Carlos III University of Madrid. In particular, we present the different activities we have designed and how we have extended SL through the design of ad-hoc objects to enhance collaborative learning in small groups, provide speech interaction with metabots, and integrate SL with Sloodle for managing collaborative sessions and multimedia contents. The proposed SL environment has been structured considering the pedagogical requirements for different subjects and includes different kinds of proposed activities: activities using the different tools provided by Sloodle, collaborative activities to construct different structures with LSL script, and debates, reinforcement exercises and tutoring using an automatic avatar providing speech interaction.

\subsection{Sloodle activities}

With regard to the course management, the utilities RegEnrol_Booth, Login Zone, Password Reset, Access Ckecker and Access Checker Door were employed to carry out the registration in the course and grant access to it only for registered students and lecturers. Several opinion surveys were developed with the Choice Horizontal tool in order to gather the opinion of the students about different aspects of the course. With respect to the contents of the course, the Presenters tool was used to project multimedia presentations from the files available in Moodle (PDF files, videos and images related to the subject). This component exploits Moodle to store and organize contents and control a streaming server to provide multimedia content through the QuickTimeTM player embedded in the SL client.

Group activities were developed with the main objective to complete glossaries by means of the MetaGloss tool, incorporating both concepts and definitions of the contents of each lesson, and the PrimDrop tool was used for the students to submit their assignments and the Vending Machine to provide resources to the students. A total of 110 activities were designed combining the functionalities provided by the Moodle server at our university (Aula Global $2^{14}$ ) and the corresponding placing and interaction in the virtual world by means of the Sloodle tools. Figure 9 shows different images corresponding to the integration of the Sloodle tools to create the educative virtual environment. The Language Processors and Formal Languages and Automata Theory courses of the Computer Science Degree at the Carlos III University of Madrid were selected for the experience. These 

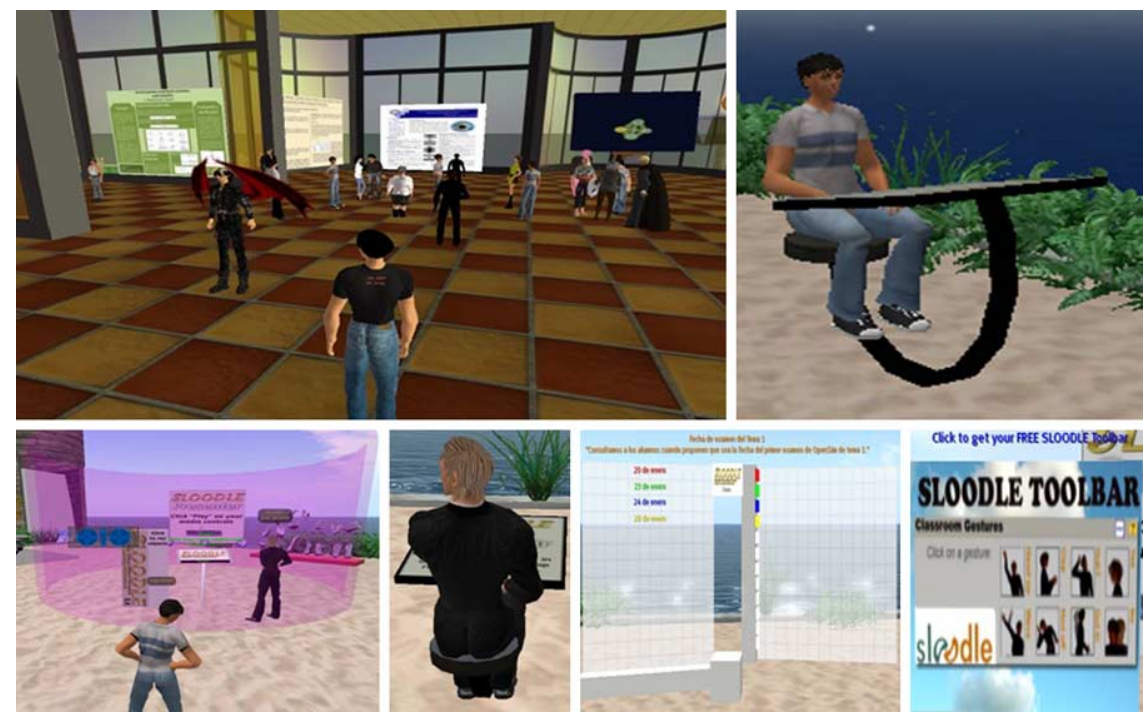

Fig. 9. Integration of the Sloodle tools to create our educative virtual environment.

subjects cover theoretical and practical contents about Finite Automata, Push-Down Automata, Turing Machines, and the design and analysis of programming languages. For the generation of the different questions and practical cases for the subject, we considered the following types of exercises:

- Questions concerning theoretical contents as a review of methodologies and concepts (e.g., what is a grammar, what is the difference between a language and a grammar?, which abstract machine recognizes Type-2 languages?, what is a token?, which are the main modules that make up a compiler? which are the main methodologies studied to develop a syntax analyser?);

- connection with programs (like the Flex lexical analyser generator, ${ }^{15}$ the Yacc parser generator, ${ }^{16}$ and the JFLAP software for experimenting with formal languages topics ${ }^{17}$ ). These programs were used to propose students to deal with practical implementations and provide them with code execution (e.g., construct a deterministic finite state automaton to recognize a specific language, define the regular expressions to detect identifiers and numbers in a specific programming language, write a parsing grammar to detect valid if-thenelse statements);

\footnotetext{
${ }^{15} \mathrm{http}: / /$ flex.sourceforge.net/

${ }^{16} \mathrm{http}: / /$ sourceforge.net/projects/byacc/

${ }^{17} \mathrm{http}: / / \mathrm{www}$.jflap.org/
}

- practical cases proposed to the student to obtain conclusions about the appropriate processes for resolve specific problems (detect reserved words in a text by means of regular expressions, recognize DNA sequences defining push-down automata, develop a practical compiler given the requirements of a simple programming language, test the application of the SLR(1) technique to parse a given input statement, apply the described optimization techniques to reduce the cost execution of a specific programming code).

\subsection{Conversational metabot}

Following the proposal described in Section 4, we have developed a conversational metabot (see Fig. 10) that facilitates two main purposes: provide academic information and carry out test and questionnaires.

For the first task, the functionalities are based on a previously developed dialogue system that worked over the telephone [14]. We defined a semantic representation in which one or more concepts represent the intention of the utterance, and a sequence of attribute-value pairs contains the information about the values given by the user. In the case of user turns, we defined four concepts related to the different queries that the user can perform to the system (Subjects, Lecturers, Doctoral studies, Registration), three task-independent concepts (Affirmation, Negation, and Not-Understood), and eight attributes (Subject-Name, Degree, Group-Name, Subject-Type, 


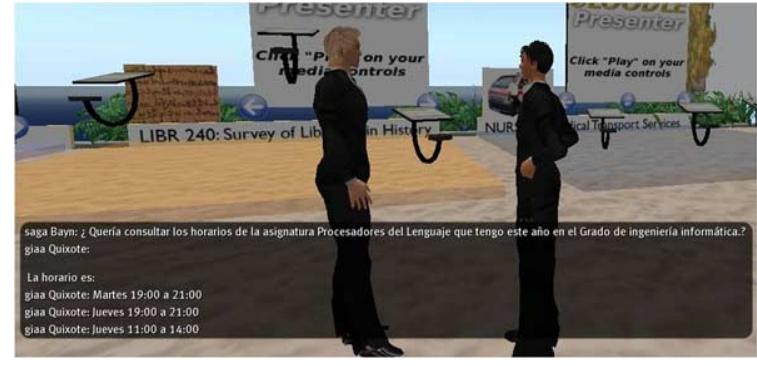

Fig. 10. Conversational metabot (left) developed to interact in virtual worlds.

Lecturer-Name, Program-Name, Semester, and Deadline). An example of the semantic interpretation of an input sentence is shown below:

User Turn: I want to know information about the subject Language Processors of Computer Science.

\section{Semantic Representation:}

(Subject)

Subject-Name: Language Processors

Degree: Computer Science

The labelling of the system turns is similar to the labelling defined for the user turns. A total of 30 taskdependent concepts were defined:

- Task-independent concepts (Affirmation, Negation, Not-Understood, New-Query, Opening, and Closing).

- Concepts used to inform the user about the result of a specific query (Subjects, Lecturers, DoctoralStudies, and Registration).

- Concepts defined to require the user the attributes that are necessary for a specific query (Subject-Name, Degree, Group-Name, SubjectType, Lecturer-Name, Program-Name, Semester, and Deadline).

- Concepts used for the confirmation of concepts (Confirmation-Subject, Confirmation-Lecturers, Confirmation-DoctoralStudies, ConfirmationRegistration) and attributes (Confirmation-SubjectName, Confirmation-Degree, ConfirmationGroupName, Confirmation-SubjectType, Confirmation-LecturerName, Confirmation-Program Name, Confirmation-Semester, and ConfirmationDeadline).

A set of 150 dialogues was acquired with the conversational metabot by means of its interaction with students and professors of our university. Figure 11 shows an example of a dialogue extracted from this corpus

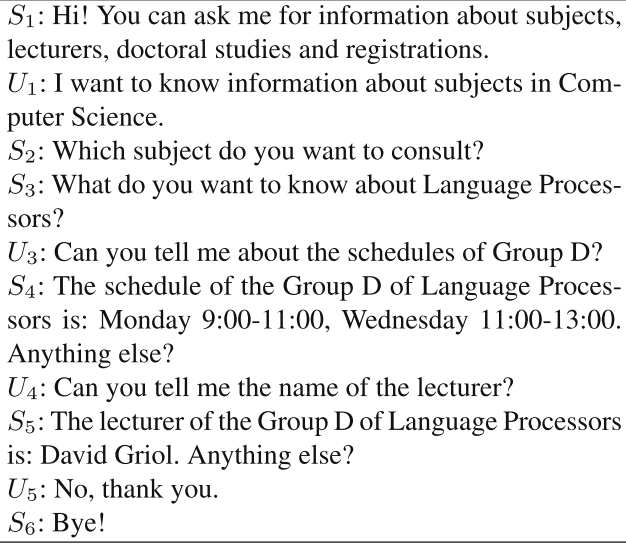

Fig. 11. Example of a dialogue acquired with the conversational metabot.

and translated from Spanish to English. Turns with S refer to system turns, and turns with $U$ refer to user turns.

The second task of the metabot was to interact with the students by presenting them a set of test questionnaires about the described subjects, including questions concerning theoretical contents as a review of methodologies and concepts. A total of 150 questionnaires were implemented using the different tools previously described for Sloodle: Presenters, Quiz Chair, Quiz Pile On, Choice Horizontal, and PrimDrop. The different questions were implemented by means of VoiceXML files. Each VoiceXML file corresponds to a specific question, includes an initial grammar, manages help events, and deliveries the student's answer to the Language Understanding module in the conversational agent.

\subsection{Manageable learning objects}

Based in other works such as [20], we developed a collection of practical activities for the development and programming of abstract entities (Finite Automata, Push-down Automata, Turing Machines) and algorithms (bottom-up syntax analysis, grammar definition and usage). The main objective was to take advantage of the graphical possibilities of the virtual worlds to clarify complex concepts that students usually find difficult to understand due to their high level of abstraction. This way, graphical simulation can be useful to illustrate their behaviour and facilitate the student learning fostering group work to collaboratively build knowledge in order to reach common predefined objectives. 


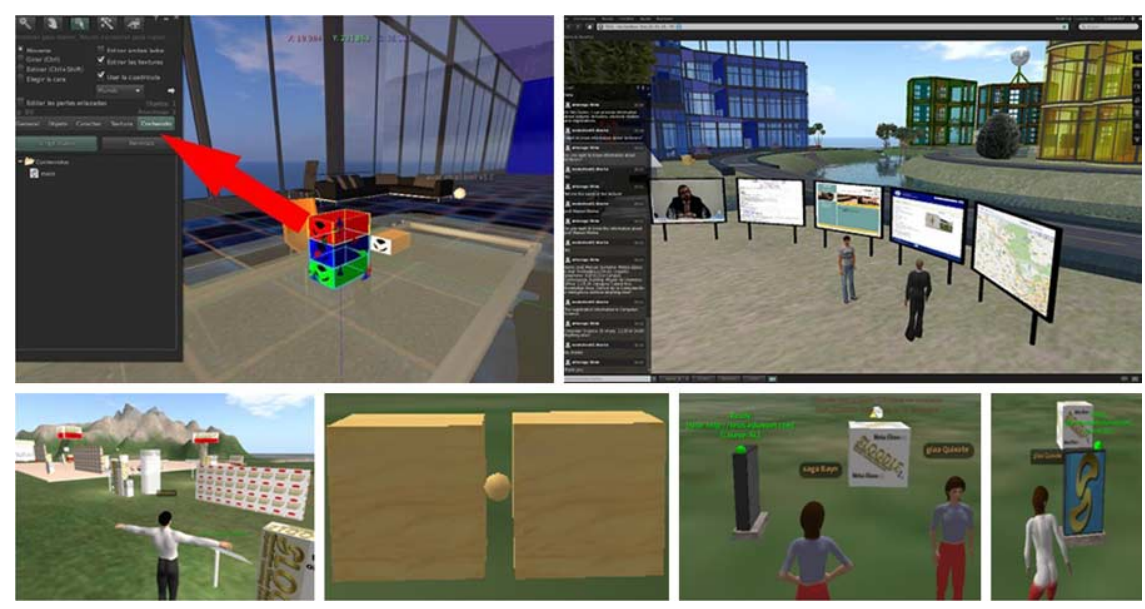

Fig. 12. Activities designed with the LSL programming language.

One of the practices consisted in the implementation of a virtual simulator of a simplified Turing Machine. The system simulates a finite state machine with a single reading header and a single tape from which bits are read sequentially one after the other. The input to the system is contained in a text file, which also contains the machine transition function. The developed Turing Machine shows step by step the intermediate values until the analysis is completed. Internally, the simulator is also a finite state machine that includes four states and the events raise the transition from one to the other. At the beginning of the simulation, the machine is in a predefined state. If the avatar offers the notecard to the machine, it accesses an initial state in which it starts analysing the inputs. At this point, the machine is listening in the control channel for a signal from one of the buttons: run or debug. When a signal arrives from any of these objects, the simulator starts working.

When the simulator stops reading, it gets into a sleepy mode in which it stays during a time interval to allow the user to read the final state of the simulation, after which the simulator detects the timeout and starts with the initial values. Figure 12 shows different images of the activities developed by the student using the LSL programming language. The images in the figure show important aspects of our proposal. The first image (right up corner) shows one of the panel of the scratch tool, which is employed to assign functionalities to the objects placed in the virtual world, as was described in Section 3. Thanks to this tool, the students can access the actions list and develop scripts with the help of an assistant. The second image (left up corner) shows sev- eral students paying attention to the instructions of the work they must develop and accessing the resources of the subjects using the Sloodle Presenter utility while they can also interact with the automatic bot, which is the avatar dressed in black in the image. In the bottom left image there is a student moving around in the virtual world to enter a work space of a subject, while the bottom center image shows the initial materials that the students had to carry out one of the proposed activities. Finally, the bottom right image shows several students interacting with a set of the objects described previously in Section 3, with the objective to develop an essay about one of the units of the subject. Active behaviours of objects have been developed with Scratch, a utility to easily create scripts in LSL previously described in Section 3.

\section{Evaluation}

The participants in the experiment were 56 students attending to the Language Processors and Formal Languages and Automata Theory described courses of the Computer Science Degree at the Carlos III University of Madrid. These subjects cover theoretical and practical contents about Finite Automata, Push-Down Automata, Turing Machines, and the design and analysis of programming languages. Each student had at his/her disposal a computer and was invited to interact with the other students and with the teacher through the virtual world.

Before the lectures started, the tutors guided the students through the virtual campus and provided them 
with a brief training session on the available communication features and avatar basic movements. The students had a basic knowledge of 2D computer graphics, and image and video editing. The teacher gave the initial lectures in a virtual classroom of the virtual campus. To perform collaborative sessions the students were divided in small groups of 2 or 3 members, each of which had at its disposal a separate environment where it was possible to discuss, visualize and record information. The conversations and decisions were automatically saved for later references in a database using the Moodle plug-in.

We have carried out a subjective evaluation through an opinion survey based on the evaluation methodologies for educative virtual environments proposed in [38], which were adapted from [60] and [34]. Three main aspects have been evaluated: perception of presence, communication and perceived sociability, and specific evaluation of the virtual environment. The responses to the questionnaire were measured on a fivepoint Likert scale ranging from 1 (for nothing/strongly disagree) to 5 (very much/strongly agree).

The questionnaire related to the evaluation of perception of presence is shown in Table 1. The questionnaire results corresponding to this measure are summarized in Table 2. As can be observed, the presence perception of the students was very high. The CF values reveal that the user perceived a good sense of control on the environment and proposed activities, as the median of all questionnaire items is 3.7. Similar results have been obtained for SF and DF, even if with the dispersion was higher.

The questionnaire related to the evaluation of communication, awareness and perceived sociability is shown in Table 3. Table 4 shows the results obtained for these questions. Also in this case, the median is high, but higher data variability denotes a heterogeneous perception of the communication features. A deeper analysis confirmed that the lower average score was obtained by the last question, which is the question concerning the avatar gestures. This indicates the students had some difficulties in communicating by using the avatar gestures. On the whole, students felt the offered communication appropriate to favour discussion and speech communication improved the interaction with other students. Let us note that, even if the median is 3.9 , a very satisfying result, there were some negative judgements by users who found it more difficult to figure out what was happening at some moments.
Table 1

Adaptation of the $[34,60]$ and [38] questionnaires for the evaluation of presence (Legend: $\mathrm{CF}=$ control factors, $\mathrm{SF}=$ sensory factors, $\mathrm{DF}=$ distraction factors, $\mathrm{RF}=$ realism factor, $\mathrm{INV}=$ involvement)

\section{Presence}

How much were you able to control events? CF

How responsive was the environment to action that you initiated (or performed)? $\mathrm{CF}$

How natural did your interactions with the environment seem? CF

How completely were all of your senses engaged? SF

How much did the visual aspects of the environment involve you? SF How much did the auditory aspects of the environment involve you? SF How natural was the mechanism that controlled movement through the environment? $\mathrm{CF}$

How aware were you of events occurring in the real world around you? DF How aware were you of your display and control devices? DF

How compelling was your sense of objects moving through space? SF

How inconsistent or disconnected was the information coming from your various senses? RF

How much did your experiences in the virtual environment seem consistent with your real-world experiences? RF, CF

Were you able to anticipate what would happen in response to the actions that you performed? CF

How completely were you able to actively survey or search the environment using vision? $\mathrm{RF}, \mathrm{CF}, \mathrm{SF}$

How well could you identify sounds? RF, SF

How well could you localize sounds? RF, SF

How well could you actively survey or search the virtual environment using speech? RF, SF

How compelling was your sense of moving around inside the virtual environment? SF

How closely were you able to examine objects? SF

How well could you examine objects from multiple viewpoints? SF

How well could you create or manipulate objects in the virtual environment? $\mathrm{CF}$

To what degree did you feel confused or disoriented at the beginning or at the end of the experimental session? RF

How involved were you in the virtual environment experience? INV

How distracting was the control mechanism?

How much delay did you experience between your actions and expected outcomes? CF

How quickly did you adjust to the virtual environment experience? $\mathrm{CF}$

How proficient in moving and interacting with the virtual environment did you feel at the end of the experience? CF

How much did the visual display quality interfere or distract you from performing assigned tasks or required activities? DF

How much did the control devices interfere with the performance of assigned tasks or with other activities? DF, CF

How well could you concentrate on the assigned tasks rather than on the mechanisms used to perform them? DF

Did you learn new techniques that enabled you to improve your performance? $\mathrm{CF}$

Were you involved in the experimental task to the extent that you lost track of time? INV

The questionnaire related to the specific evaluation of the virtual environment is shown in Table 5. In Table 6 we summarize the results corresponding to this evaluation and the results concerning the additional questions referring to the perception of learning and global satisfaction. As can be observed, the mean of all the items of this category is high (4.5) and the low standard deviation indicates that most subjects agreed 
Table 2

Results related to the perception of presence

\begin{tabular}{lcccccc}
\hline & Total & Scaled & \multicolumn{4}{c}{ Factors } \\
\cline { 4 - 7 } & & & CF & SF & DF & RF \\
\hline Mean Score & 117.2 & 3.7 & 3.9 & 3.7 & 3.9 & 3.4 \\
Std. Deviation & 9.8 & & 0.5 & 1.1 & 0.8 & 0.6 \\
Maximum value & 147.0 & 4.6 & 5 & 5 & 5 & 5 \\
Minimum Value & 89 & 2.8 & 4 & 3 & 3 & 2 \\
\hline
\end{tabular}

Table 3

Adaptation of the [34,60] and [38] questionnaires for the evaluation of communication, awareness and perceived sociability (Legend: $\mathrm{COM}=$ communication, $\mathrm{AW}=$ awareness, $\mathrm{PS}=$ perceived sociability)

Communication, awareness and perceived sociability

Communicating with the other participants was easy COM

The system increased the opportunity of discussing with the others COM

Conversation has been properly managed COM

Non-verbal communication (gesture) was adequate COM

I have been immediately aware of the existence of the other participants AW

I was aware of what was going on AW

I was aware of the participant roles (teacher, tutor, student) AW

This environment enabled me to easily contact my team-mates AW

I did not feel lonely in this environment AW

This environment enabled me to get a good impression of my team-mates PS

This environment allows spontaneous informal conversations PS

This environment allowed for non-task-related conversations PS

This environment enabled me to make close friendships with my team-mates PS

Table 4

Results related to communication, awareness and perceived sociability

\begin{tabular}{lccl}
\hline & COM & AW & PS \\
\hline Mean Score & 4.1 & 3.9 & 3.8 \\
Std. Deviation & 1.3 & 1.1 & 1.0 \\
Maximum value & 5 & 5 & 4 \\
Minimum Value & 2 & 3 & 3 \\
\hline
\end{tabular}

in assigning a very positive score to the activities in the virtual environment and were satisfied with the experience.

We have also completed an objective evaluation of the conversational metabot considering the following statistical measures:

1. Dialogue success rate (Success Rate). This is the percentage of successfully completed dialogues in which the metabot provides the correct information to each one of the required questions.

2. Average number of turns per dialogue (nT).

3. Confirmation rate (Confirmation Rate). It was computed as the ratio between the number of ex-
Table 5

Adaptation of the $[34,60]$ and [38] questionnaires for the specific evaluation of the virtual environment

\section{Virtual environment}

The environment design was stimulating

The object metaphors were intuitive

Objects reacted in an inconsistent/consistent way to selection and manipulation

The User Interface components, needed to participate, were easy to locate

Amount of information that was displayed on the screen was adequate

Arrangement of information on the screen was logical

The design of the didactical environments was logical

This environment enabled me to learn

I am satisfied with the experience

Table 6

Results related to the specific evaluation of the virtual environment

\begin{tabular}{lccc}
\hline & Virtual Environment & Learning & Satisfaction \\
\hline Mean Score & 4.5 & 4.4 & 4.7 \\
Std. Deviation & 0.4 & 0.9 & 0.5 \\
Maximum value & 3 & 2 & 3 \\
Minimum Value & 5 & 5 & 5 \\
\hline
\end{tabular}

plicit confirmations turns (nCT) and the number of turns in the dialogue (nCT/nT).

4. Average number of corrected errors per dialogue (nCE). This is the average of errors detected and corrected by the dialogue manager of the conversational metabot. We have considered only those errors that modify the values of the attributes and that could cause the failure of the dialogue.

5. Average number of uncorrected errors per dialogue (nNCE). This is the average of errors not corrected by the dialogue manager. Again, only errors that modify the values of the attributes are considered.

6. Error correction rate (ECR). The percentage of corrected errors, computed as nCE/ $(\mathrm{nCE}+$ nNCE).

The results presented in Table 7 for the described 150 dialogues show that the developed conversational metabot could interact correctly with the users in most cases, achieving a success rate of $94 \%$. The dialogue success depends on whether the system provides the correct data for every query required by the user. The analysis of the main problems detected in the acquired dialogues shows that, in some cases, the system did not detect that the user wanted to finish the dialogue. A second problem was related to the introduction of data with a high confidence value due to errors generated by the automatic speech recognizer that were not detected. However, the evaluation confirms a good oper- 


\begin{tabular}{lcccccc}
\hline & Success Rate & nT & Confirmation Rate & ECR & nCE & nNCE \\
\hline Conversational Metabot & $94 \%$ & 11.6 & $28 \%$ & $93 \%$ & 0.89 & 0.06 \\
\hline
\end{tabular}

Table 8

Results of the subjective evaluation of the conversational metabot

\begin{tabular}{lllllllllll}
\hline & Q1 & Q2 & Q3 & Q4 & Q5 & Q6 & Q7 & Q8 & Q9 & Q10 \\
\hline Mean Score & 4.6 & 2.4 & 4.0 & 4.4 & 4.2 & 3.3 & 3.0 & 2.9 & 2.6 & 4.4 \\
Maximum Value & 5 & 3 & 4 & 5 & 5 & 4 & 4 & 4 & 4 & 5 \\
Minimal Value & 4 & 1 & 3 & 3 & 3 & 2 & 2 & 2 & 1 & 3 \\
Std. Deviation & 0.3 & 1.7 & 0.6 & 0.5 & 0.3 & 0.5 & 1.1 & 1.0 & 1.3 & 0.6
\end{tabular}

ation of the approach since the information is correctly provided by the metabot in the majority of cases, as it is also shown in the value of the error correction rate.

In addition, we have completed an evaluation of the conversational metabot based on questionnaire to assess the students' subjective opinion about the metabot performance. The questionnaire had 10 questions and the answers were placed in a 5-points Likert scale: i) $Q 1$ : State on a scale from 1 to 5 your previous knowledge about new technologies for information access; ii) $Q 2$ : State on a scale from 1 to 5 your previous experience with virtual worlds like Second Life; iii) $Q 3$ : How well did the metabot understand you?; iv) $Q 4$ : How well did you understand the messages generated by the metabot?; v) $Q 5$ : Was it easy for you to get the requested information?; vi) $Q 6$ : Was the interaction rate adequate?; vii) $Q 7$ : Was it easy for you to correct the metabot errors?; viii) $Q 8$ : Were you sure about what to say to the system at every moment?; ix) $Q 9$ : Do you believe the system behaved similarly as a human would do?; x) Q10: In general terms, are you satisfied with the metabot performance? Table 8 shows the average, minimal and maximum values for the subjective evaluation.

From the results of the evaluation, it can be observed that students positively evaluated the facility of obtaining the data necessary to complete the exercises and found the interaction rate suitable. The suggestions that they mentioned for the improvement of the system include the correction of system errors and a better clarification of the set of actions expected by the metabot at each time. Another interesting consideration concerns the correlation between the student background and the rest of scores. We verified that the questionnaire results are not influenced by the sample characteristics: user impressions are positive also when students did not have a previous experience with virtual worlds. The students were very satisfied with the experience, not only because it facilitated learning but also because it was amusing for them. Additionally, the marks improved with respect to previous years in which the virtual environment was not used (2011: average mark of 4.05 and $53.85 \%$ of students passed the subject in the first examination session; 2012: average mark of 5.92 and $65.35 \%$ of students passed the subject in the first examination session).

The students were very positive about the experience, and highlighted the fact that the use of the virtual environment made them enjoy the subjects and have fun while learning, and that it was easier for them to understand the contents of the subjects, as the virtual activities reinforced the concepts studied in class. They also reported that the activities fostered their abilities to work collaboratively and share ideas with the peer students.

Although the students liked the amount of options and tools that they had at their disposal to work in the virtual world, for some of them it had a negative effect as sometimes they felt disoriented. This also happened to the students who were used to play virtual video games, as they follow a script which did not exist in the subject, where the student chooses his/her behaviour and the outcomes were not predefined. Despite these minor negative impressions, a majority of students said that they would repeat the experience for other courses.

Also the teaching staff that participated in the experience reported very positive impressions, even when initially they were reluctant to use virtual worlds. Specially, they highlighted the fact that students seemed to be more engaged in learning, and had a more significant learning when carrying out such a rich variety of activities in the classroom, laboratory and the virtual environment. On the other hand, most of them suggested that it would be desirable to create some repository in which they could share the activities created, as well as a description of the experiences.

\section{Conclusions and future work}

Immersive Virtual Worlds in combination with Artificial Intelligence techniques and Natural Language Processing bring a wide set of opportunities that can be exploited for creating intelligent learning environ- 
ments, as they provide an enormous range of possibilities for evaluating new learning scenarios in which the students can explore, meet other residents, socialize, participate in individual and group activities, and create and trade virtual objects and services with one another.

In this paper, we have proposed a model to develop such intelligent environments over Second Life and OpenSimulator based on the use of the Moodle Learning Management System, the development of programmable objects, and the development of enhanced embodied conversational metabots, thus complementing the state-of-the-art approaches which are usually based solely on visual information. A practical implementation of the model of several Computer Science subjects in our University has been presented and evaluated, obtaining very satisfactory results in terms of presence, commitment, performance and satisfaction.

The introduction of new technologies in education requires adequate training for the teachers. In the particular case of our evaluation, the lecturers and students implied had a strong technical background. However, as described in Section 2, virtual worlds and Sloodle have been successfully used by teachers of different levels and with different degrees of technical knowledge. With respect to our proposal, we have worked in rapid prototyping of conversational agents by non-technical users [25], and are currently working to employ our last advancements to make the architecture totally transparent for the teachers that will use, so that they just have to supply a simple model for the conversations expected.

As a future work we intend to study the differences between the student models generated in this paper and the ones that could be obtained from real interactions in the classroom. This way, we plan to study the similarities and differences in the behaviour of the students when influenced by the image of their avatar compared to their usual conversational behaviour. Additionally, we want to carry out a more detailed study on the effect of emotional behaviour of metabots by integrating an emotion recognizer in the system to improve the communication between the system and the bots and avoid negative emotions that can influence the learning process such as frustration.

The practical application of our proposal has not been validated with students with motor and visual disabilities. However, this is for us one of the main objectives of introducing speech interaction to facilitate a more natural human-machine communication and eliminate the barriers that difficult the access to new technologies. In addition, the integration of mobile devices is also very interesting and their use in education is receiving increasing attention from the research community. We plan to investigate the use of tablets in combination with our proposal as an m-learning experience. ${ }^{18}$ We are particularly interested in studying what type of devices are better suited for this purpose, as intelligent phones may not be a good option provided the computational and graphical requirements of virtual worlds.

In addition, we are extending the experience during this academic year, including in our study several functionalities to adapt the environment and Sloodle tools taking into account student's specific needs, considering their evolution during the course as one of the main aspects to perform this adaptation. We are also evaluating the benefits of using the Sloodle tools in combination with open-source metaverses like the ones that can be created using OpenSimulator and the OSgrid Project.

\section{Acknowledgements}

This work was supported in part by Projects MINECO TEC2012-37832-C02-01, CICYT TEC201128626-C02-02, CAM CONTEXTS (S2009/TIC-1485).

\section{References}

[1] C. Aldrich, Learning by Doing: A Comprehensive Guide to Simulations, Computer Games, and Pedagogy in e-Learning and Other Educational Experiences, Pfeiffer, 2005.

[2] A. Andrade, A. Bagri, K. Zaw, B.A. Roos, and J.G. Ruiz, Avatar-mediated training in the delivery of bad news in a virtual world, Journal of Palliative Medicine 13(12) (2010), 1-14, Mary Ann Liebert Inc.

[3] K. Andreas, T. Tsiatsos, T. Terzidou, and A. Pomportsis, Fostering collaborative learning in Second Life: Metaphors and affordances, Computers \& Education 55(2) (2010), 603-615, Elsevier.

[4] P. Anthamatten and S.S. Ziegler, Teaching geography with 3-D visualization technology, Journal of Geography 105(6) (2006), 231-237, Taylor \& Francis.

[5] A. Arroyo, F. Serradilla, and O. Calvo, Multimodal agents in Second Life and the new agents of virtual 3D environments, in: Proc. IWINAC'09, 2009, pp. 506-516.

[6] R.K. Atkinson, S.J. Derry, A. Renkl, and D.W. Wortham, Learning from examples: Instructional principles from the worked examples research, Review of Educational Research

${ }^{18}$ http://www.m-learning.org/ 
70 (2000), 181-214, American Educational Research Association.

[7] J.C. Augusto and H. Aghajan, Thematic issue: Virtual and mixed reality intelligent environments (preface), Journal of Ambient Intelligence and Smart Environments 4(1) (2012), 11 , IOS Press.

[8] R. Aylett and M. Cavazza, Intelligent virtual environments: A state-of-the-art report, in: Proc. Eurographics 2001, STAR Reports, 2001, pp. 87-109.

[9] J.N. Bailenson, N. Yee, J. Blascovich, A.C. Beall, N. Lundblad, and M. Jin, The use of immersive virtual reality in the learning sciences: Digital transformations of teachers, students, and social context, Journal of the Learning Sciences 17(1) (2008), 102-141, Taylor \& Francis

[10] F. Bailey and M. Moar, The vertex project: Children creating and populating 3D virtual worlds, Journal of Art and Design Education 20(1) (2001), 19-30, John Wiley \& Sons.

[11] C. Bakas and T. Mikropoulos, Design of virtual environments for the comprehension of planetary phenomena based on students' ideas, International Journal of Science Education 25(8) (2003), 949-967, Taylor \& Francis.

[12] S. Barab, T. Sadler, C. Heiselt, D. Hickey, and S. Zuiker, Relating narrative, inquiry, and inscriptions: Supporting consequential play, Journal of Science Education and Technology 16(1) (2007), 59-82, Springer.

[13] J. Barkand and J. Kush. GEARS a 3D virtual learning environment and virtual social and educational world used in online secondary schools, Electronic Journal of e-Learning 7(3) (2009), 215-224, Academic Publishing Limited.

[14] Z. Callejas and R. López-Cózar, Relations between de-facto criteria in the evaluation of a spoken dialogue system, Speech Communication 50(8-9) (2008), 646-665, Elsevier.

[15] M. Cavazza, R.S. de la Cámara, and M. Turunen, How was your day? A companion ECA, in: Proc. AAMAS'10, 2010, pp. 1629-1630.

[16] M. Davies and V. Callaghan, iWorlds: Generating artificial control systems for simulated humans using virtual worlds and intelligent environments. Journal of Ambient Intelligence and Smart Environments 4(1) (2012), 1-23, IOS Press.

[17] K. Ellison and C. Matthews, Virtual history: A socially networked pedagogy of Enlightenment, Educational Research 52(3) (2010), 297-307, Taylor \& Francis.

[18] L. Fryer and R. Carpenter, Bots as language learning tools, Language Learning and Technology 10(3) (2006), 8-14, NFLRC and CLEAR.

[19] M. Gardner and B. Horan, Using virtual worlds for online role-play, in: Proc. 1st European Immersive Education Summit (iED'11), 2011, pp. 1-6

[20] G. De Gasperis, L. Di Maio, T. Di Mascio, and F. Niva, Il Metaverso Open Source: Strumento Didattico per Facoltà Umanistiche, in: Proc. Didamatica, 2011, pp. 1-10.

[21] C. Girvan and T. Savage, Identifying an appropriate pedagogy for virtual worlds: A Communal Constructivism case study, Computers \& Education 55(1) (2010), 342-349, Elsevier

[22] E. Glaserfeld, Constructivism in Education, Pergamon Press, 1989.

[23] D. Griol and J.M. Molina, Using virtual worlds and Sloodle to develop educative applications, in: International Workshop on Evidence-Based Technology Enhanced Learning, Advances in Intelligent and Soft Computing, Vol. 152, Springer, 2012, pp. 99-106.
[24] D. Griol, L. Hurtado, E. Segarra, and E. Sanchis, A statistical approach to spoken dialog systems design and evaluation, Speech Communication 50(8-9) (2008), 666-682, Elsevier.

[25] D. Griol, J.M. Molina, and Z. Callejas, Bringing together commercial and academic perspectives for the development of intelligent AmI interfaces, Journal of Ambient Intelligence and Smart Environments 4(3) (2012), 83-207, IOS Press.

[26] T. Heinroth and W. Minker, Introducing Spoken Dialogue Systems into Intelligent Environments, Springer, 2013.

[27] L. Jarmon, T. Traphagan, M. Mayrath, and A. Trivedi, Virtual world teaching, experiential learning, and assessment: An interdisciplinary communication course in Second Life, Computers \& Education 53(1) (2009), 169-182, Elsevier.

[28] K. Jauregi, S. Canto, R. Graaff, T. Koenraad, and M. Moonen, Verbal interaction in Second Life: Towards a pedagogic framework for task design, Computer Assisted Language Learning Journal 24(1) (2011), 77-101, Taylor \& Francis.

[29] H. Kanematsu, Y. Fukumura, D.M. Barry, S.Y. Sohn, and R. Taguchi, Multilingual discussion in metaverse among students from the USA, Korea and Japan, in: Lecture Notes in Computer Science, Vol. 6279, Springer, 2010, pp. 200209.

[30] S. Kartakis, A design-and-play approach to accesible user interface development in Ambient Intelligence Environments, Journal Computers in Industry 61(4) (2010), 318-328, Elsevier.

[31] A. Kerly, R. Ellis, and S. Bull, Conversational agents in elearning, in: Proc. AI'08, 2008, pp. 169-182.

[32] D.J. Ketelhut, C. Dede, J. Clarke, B. Nelson, and C. Bowman, Assessment of problem solving using simulations, in: Studying Situated Learning in a Multi-User Virtual Environment, E. Baker, J. Dickieson, W. Wulfeck \& H. O’Neil, eds, Lawrence Erlbaum Associates, 2007.

[33] D.J. Ketelhut, C. Dede, J. Clarke, B. Nelson, and C. Bowman, Multimodal dialogue for ambient intelligence and smart environments, in: Handbook of Ambient Intelligence and Smart Environments, H. Nakashima, H. Aghajan and J.C. Augusto, eds, Springer, 2010.

[34] K. Kreijns, P.A. Kirschner, W. Jochems, and H. van Buuren, Measuring perceived sociability of computer-supported collaborative learning environments, Computers \& Education 49 (2007), 176-192, Elsevier.

[35] E.A.-L. Lee, K.W. Wong, and C.C. Fung, How does desktop virtual reality enhance learning outcomes? A structural equation modeling approach, Computers \& Education 55(4) (2010), 1424-1442, Elsevier.

[36] M.B. Ligorio, A. Talamo, and R.S. Simons, Euroland: A virtual world fostering collaborative learning at a distance, in: Proc. First Research Workshop of EDEN Research and Innovation in Open and Distance Learning, 2000.

[37] M. Limniou, D. Roberts, and N. Papadopoulos, Full immersive virtual environment CAVETM in chemistry education, Com puters \& Education 51(2) (2008), 584-593, Elsevier.

[38] A. De Lucia, R. Francese, I. Passero, and G. Tortora, Development and evaluation of a virtual campus on Second Life: The case of SecondDMI, Computers \& Education 52 (2009), 220 233, Elsevier.

[39] A.D. Lucia, R. Francese, I. Passero, and G. Tortora, Development and evaluation of a virtual campus on Second Life: The case of SecondDMI, Computers \& Education 52(1) (2009), 220-233, Elsevier. 
[40] R. López-Cózar and M. Araki, Spoken, Multilingual and Multimodal Dialogue Systems: Development and Assessment, Wiley, 2005.

[41] M.F. McTear, Spoken Dialogue Technology: Towards the Conversational User Interface, Springer, 2004.

[42] T.A. Mikropoulos and A. Natsis, Educational virtual environments: A ten-year review of empirical research (1999-2009), Computers \& Education 56(3) (2011), 769-780, Elsevier.

[43] W. Minker, M. Weber, H. Hagras, V. Callagan, and A. Kameas, eds, Advanced Intelligent Environments, Springer, 2004.

[44] W. Minker, R. López-Cózar, and M.F. McTear, The role of spoken language dialogue interaction in Intelligent Environments, Journal of Ambient Intelligence and Smart Environments 1(1) (2009), 31-36, IOS Press.

[45] H. Park, The effect of activities in virtual worlds as a communication environment to understand each other, Journal of Cyber Therapy and Rehabilitation 3(1) (2010), 71-82, Virtual Reality Medical Institute.

[46] S.J. Perry and I. Bulatov, The influence of new tools in virtual learning environments on the teaching and learning process in chemical engineering, Chemical Engineering Transactions 21 (2010), 1051-1056, The Italian Association of Chemical Engineering.

[47] S. Persky, Employing immersive virtual environments for innovative experiments in health care communication, Patient Education and Counseling Journal 82(3) (2011), 313-317, Elsevier.

[48] A. Petrakou, Interacting through avatars: Virtual worlds as a context for online education, Computers \& Education 54(4) (2010), 1020-1027, Elsevier.

[49] R. Pieraccini and L.R. Rabiner, The Voice in the Machine: Building Computers That Understand Speech, MIT Press, 2012.

[50] H. Pon-Barry, K. Schultz, E.O. Bratt, B. Clark, and S. Peters, Responding to student uncertainty in spoken tutorial dialogue systems, International Journal of Artificial Intelligence in Education 16 (2006), 171-194, IAIED Constitution.
[51] C.M. Reigeluth ed., Instructional-Design Theories and Models: A New Paradigm of Instructional Theory, Vol. II, Routledge, 1999.

[52] M. Rico, D. Camacho, X. Alamán, and E. Pulido, A high school educational platform based on virtual worlds, in: Proc. 2nd Workshop on Methods and Cases in Computing Education, 2009.

[53] M. Rymaszewski, W.J. Au, C. Ondrejka, R. Platel, S. Van gorden, J. Cézanne, P. Cézanne, B. Batstone-Cunningham, A. Krotoski, C. Trollop, and J. Rossignol, Second Life: The Official Guide, Sybex, 2008.

[54] S. Santoveña and T. Feliz, El Proyecto added value of teaching in a virtual world (AVATAR), Didáctica, Innovación y Multimedia 18 (2010), 1-9, Red DIM.

[55] R.C. Schank, Designing World-Class E-Learning: How IBM, GE, Harvard Business School and Columbia University Are Succeeding at E-Learning, McGraw-Hill, 2002.

[56] R.C. Schank, What we learn when we learn by doing, Technical report, Northwestern University, Institute for Learning Sciences, 2005

[57] C. Vaquero, O. Saz, E. Lleida, J. Marcos, and C. Canalís, VOCALIZA: An application for computer-aided speech therapy in Spanish language, in: Proc. IV Jornadas en Tecnología del Habla, 2006

[58] Y. Wang, W. Wang, and C. Huang, Enhanced semantic question answering system for e-learning environment. in: Proc. AINAW'07, 2007.

[59] W. Winn, F. Stahr, C. Sarason, R. Fruland, P. Oppenheimer, and Y.-L. Lee, Learning oceanography from a computer simulation compared with direct experience at sea, Journal of Research in Science Teaching 43(1) (2006), 25-42, John Wiley \& Sons.

[60] B.G. Witmer and M.J. Singer, Measuring presence in virtual environments: A presence questionnaire, Presence: Teleoperators and Virtual Environments 7(3) (1998), 225-240, MIT Press. 